\title{
Mechanical Properties of Ag Nanoparticle Thin Films Synthesized by Supersonic Cluster Beam Deposition
}

Simone Peli ${ }^{1}$, Emanuele Cavaliere ${ }^{1}$, Giulio Benetti, ${ }^{1,2}$ Marco Gandolfi, ${ }^{1,3}$, Mirco Chiodi ${ }^{4}$, Claudia Cancellieri ${ }^{4}$, Claudio Giannetti ${ }^{1}$, Gabriele Ferrini ${ }^{1}$, Luca Gavioli ${ }^{1}$, Francesco Banfi ${ }^{1}$

${ }^{1}$ Interdisciplinary Laboratories for Advanced Materials Physics (i-LAMP) and Dipartimento di Matematica e Fisica, Università Cattolica del Sacro

Cuore, Via Musei 41, 25121 Brescia

${ }^{2}$ Laboratory of Solid state Physics and Magnetism, Department of Physics and Astronomy, KU Leuven, Celestijnenlaan 200D, B-3001, Leuven, Belgium

${ }^{3}$ Laboratory of Soft Matter and Biophysics, Department of Physics and Astronomy, KU Leuven, Celestijnenlaan 200D, B-3001 Leuven, Belgium

${ }^{4}$ Empa, Swiss Federal Laboratories for Materials Science and Technology, Laboratory for Joining and Interface Technology, Überlandstrasse 129, 8600 Dübendorf, Switzerland

E-mail: luca.gavioli@unicatt.it; francesco.banfi@unicatt.it

\section{Supporting Information}




\section{X-Ray measurements geometrical configurations}

Two different diffractometer geometries were used during X-ray diffraction measurements. For Grazing-Incidence X-ray Diffraction (GIXD) a fixed incident angle $\omega$ was chosen and maintained throughout the entire $2 \theta$ scan (see Figure S1-a)). For X-Ray Reflectivity (XRR) and stress measurements, a Bragg-Brentano configuration was used. In this case the incident angle $\omega$ was varied to keep the Bragg condition at every angle during the scan (i.e. $\omega=$ $2 \theta / 2$ ). For the stress measurements, the same scan was repeated at different tilt angles $\psi$ with respect to the pristine diffraction plane (see Figure S1-b)).
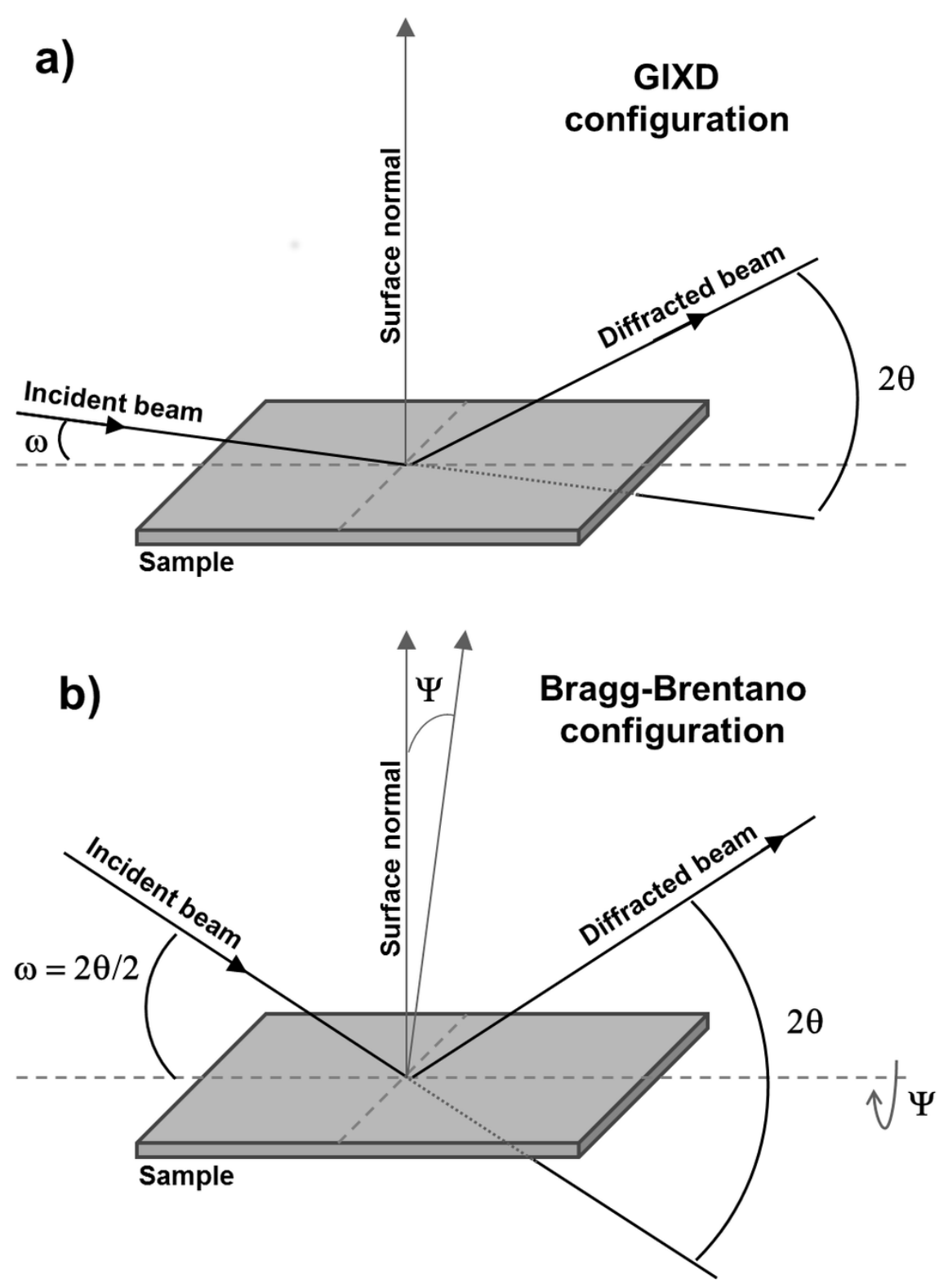

Figure S1. Schemes of the different diffractometer geometries used for (a) GIXD (b) XRR and Stress measurements. 


\section{X-Ray Reflectivity}

One of the most commonly used direct techniques for density determination is X-Ray Reflectivity (XRR), in which an X-ray beam irradiates the sample at a grazing angle $\omega$ and the reflected intensity is measured at a corresponding angle of $2 \theta$. In the present case, $\omega=$ 20/2, so-called Bragg-Brentano mode or specular reflection mode.

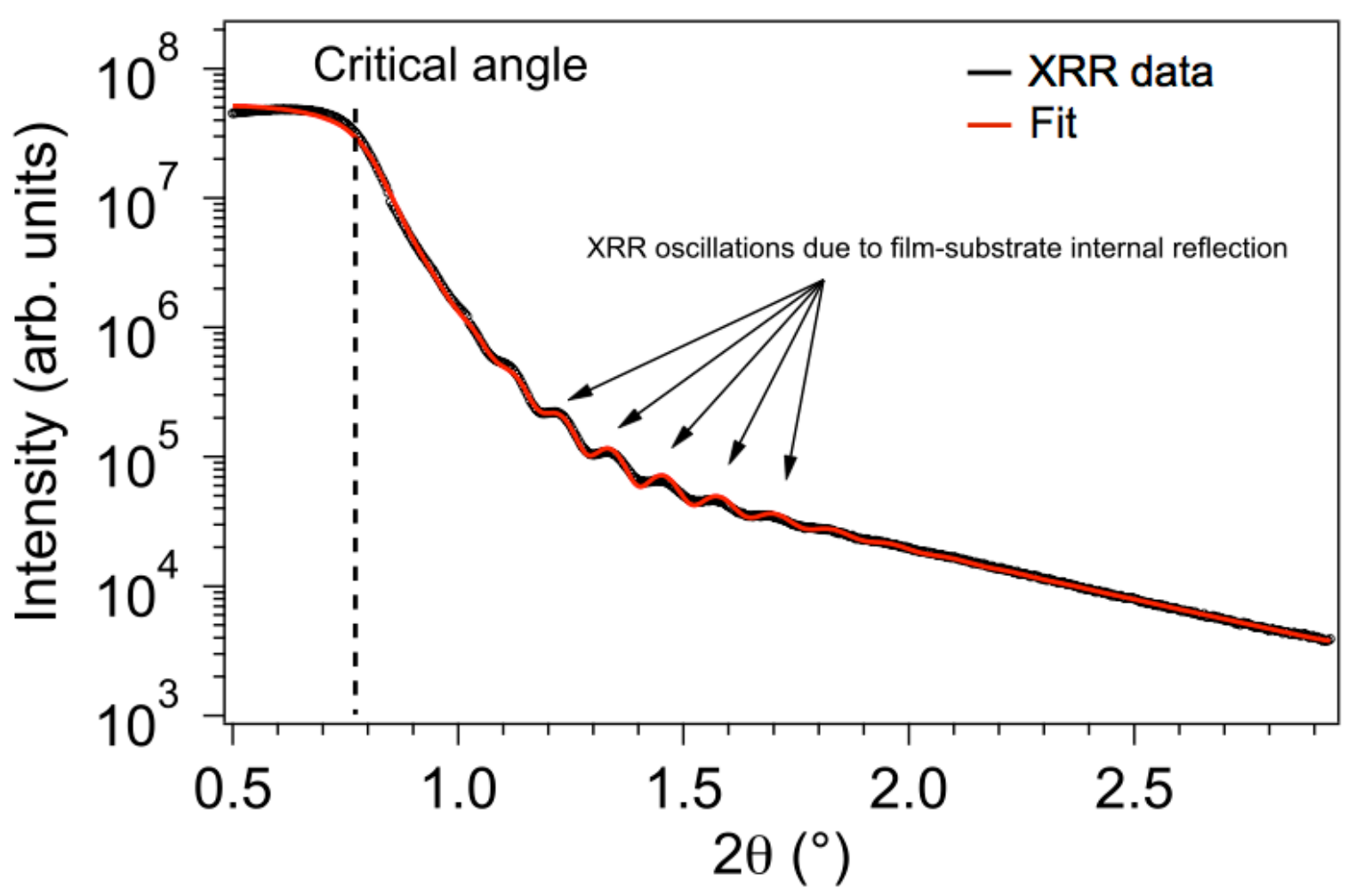

Figure S2. X-Ray Reflectivity measurement carried out on the Ag NP film (black) and fit (red curve).

The reflection signal, see black line in Figure S2, is due to the differences in the electron densities in the various layers present in the sample. For incident angles $\omega$ below the critical angle, total external reflection occurs (the reflectivity intensity shows a plateau). When $\omega$ is higher than the critical angle, the interference between the X-rays reflected at topmost surface and those reflected at the interface between the film and the substrate give rise to XRR oscillations. The period of such oscillations is related to the thickness of the film, while the value of the critical angle is primarily determined by the density of the top layer (in our case the NP film density). The estimation of the critical angle usually involves the determination of the angle at which the reflected intensity is $50 \%$ of the intensity in plateau. In our case, the estimation gives $0.39^{\circ}$, corresponding to a density of $8450 \mathrm{~kg} / \mathrm{m}^{3}$, much lower than the Ag bulk density of $10490 \mathrm{~kg} / \mathrm{m}^{3}$. 


\section{Grazing Incidence X-Ray Diffraction}

Grazing-Incidence X-ray Diffraction (GIXD) measurements were carried out ex-situ on the NP film with an incident angle $\omega$ of $1.2^{\circ}$ (see Figure S3). In this configuration, the incident angle $\omega$ (between the X-ray beam and the surface of the sample) is kept now constant while the detection angle $2 \theta$ is varied during the acquisition. The grazing-incidence geometry allows maximizing the signal coming from the topmost layer. Thus, no contribution of the substrate is detected and only peaks ascribed to polycrystalline Ag are found. The profiles of the two most intense reflections (i.e. (111) and (200)) were fitted using Gaussian peaks to estimate their FWHMs $\left(\beta_{\{111\}}\right.$ and $\left.\beta_{\{200\}}\right)$. After subtracting the instrumental broadening $\left(\mathrm{FWHM}=0.04^{\circ}\right)$, the coherency length corresponding to the two sets of planes $\{111\}$ and $\{200\}$ was retrieved using the Scherrer formula. The two estimations were then averaged giving a NPs total coherency length - indicative of grain size - of $6.5 \pm 0.5 \mathrm{~nm}$.

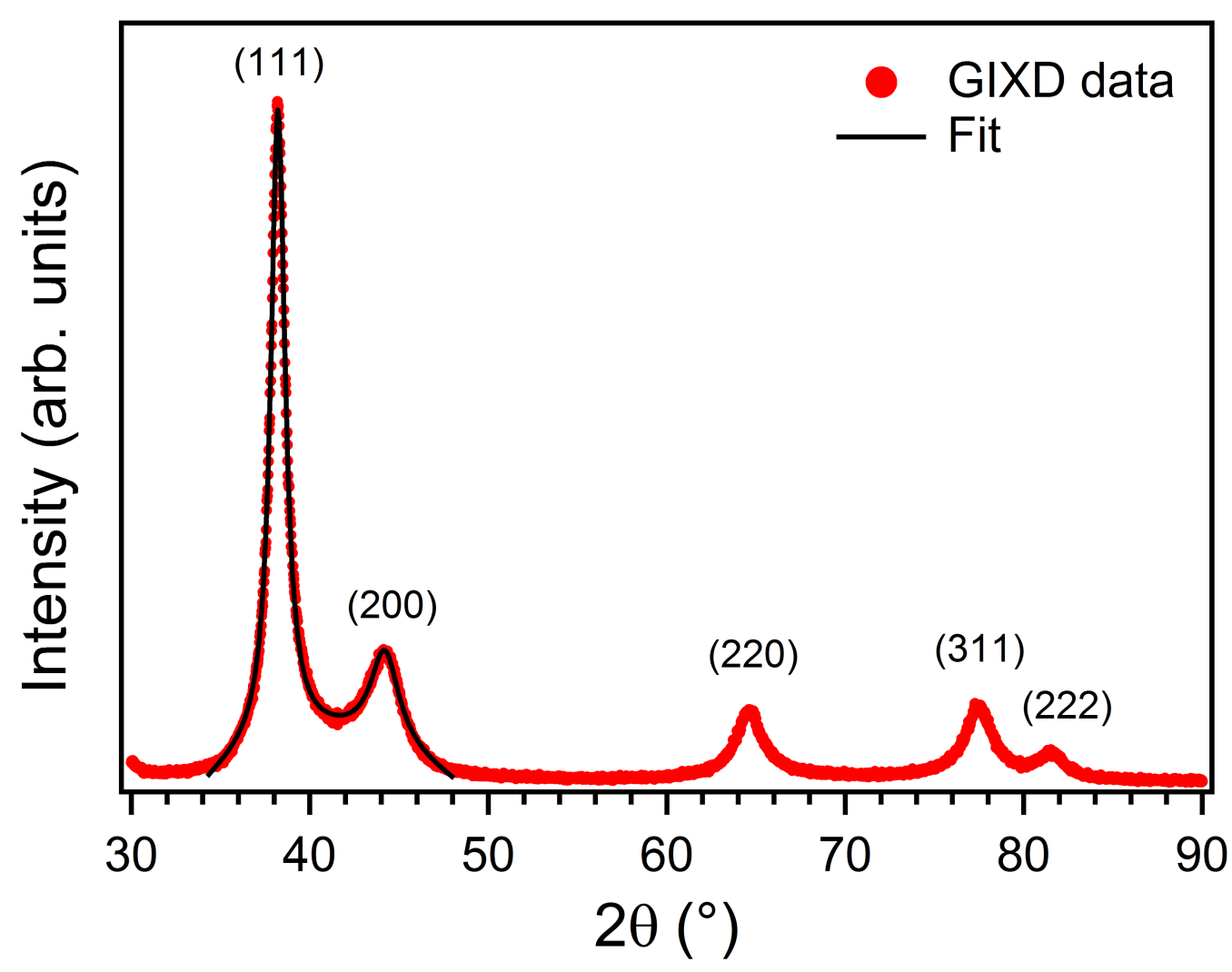

Figure S3. Grazing-incidence X-Ray diffraction taken on the Ag NP film. The reflections ascribed to polycrystalline $\mathrm{Ag}$ are reported together with the fit used to estimate the average grain size of the NPs. 


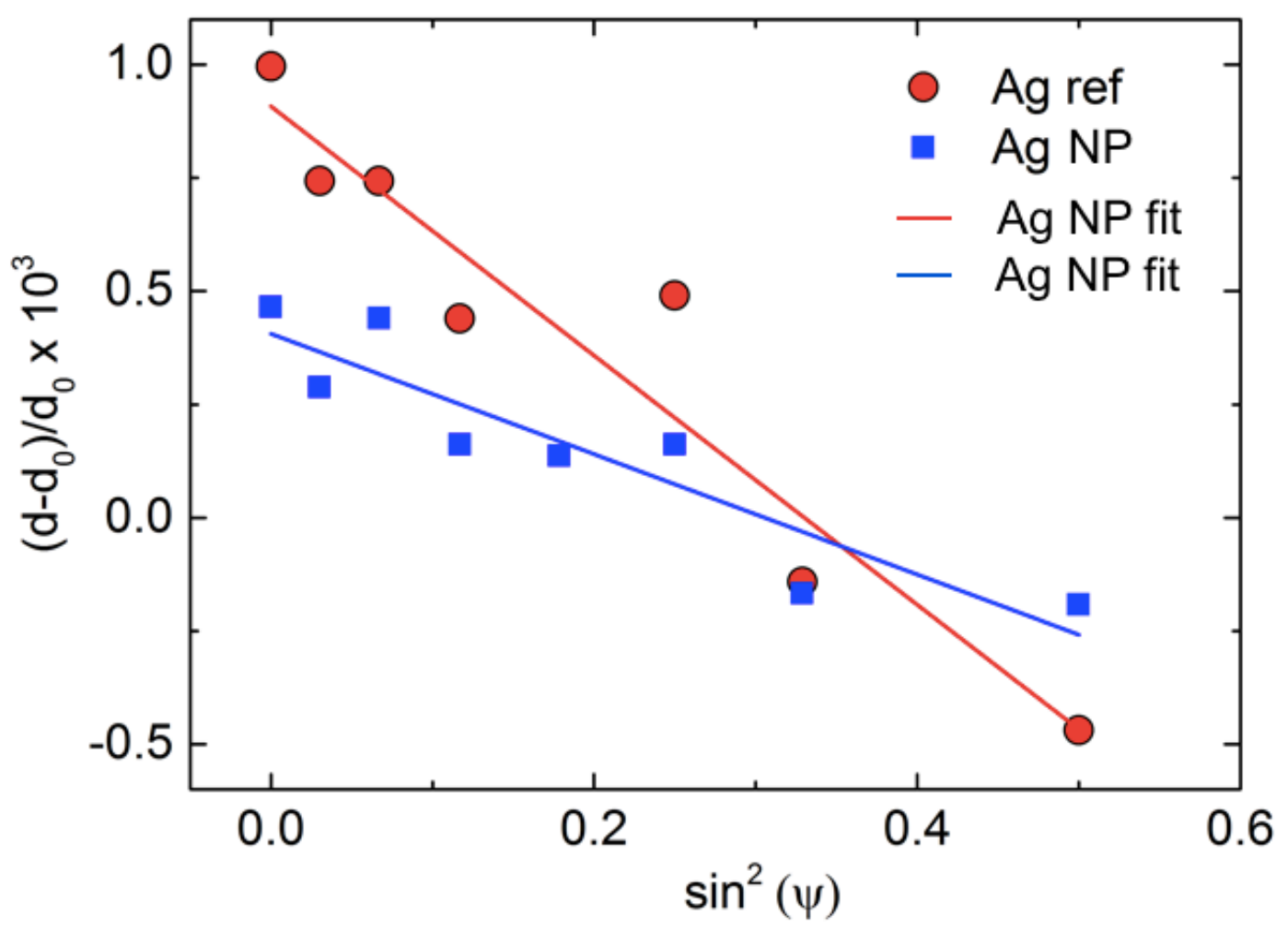

Figure S4. Plots of the strain as a function of $\sin ^{2}(\psi)$ as measured on the Ag reference layer (red dots) and the Ag NP film (blue squares). The lines are linear fits to the data.

Residual stress in the Nanoparticles (NP) was investigated by a Bruker Discover (D8) diffraction system equipped with a focal point configuration.

The residual stress analysis is carried out using the so-called " $\sin ^{2} \psi$-method" suitable for polycrystalline samples (without any preferential orientation), based on the variation of the diffraction peak position as a function of $\sin ^{2} \psi, \psi$ being the tilt angle. The most intense $\operatorname{Ag}(111)$ reflection was chosen for this study. A straight line is expected in the plot of the strain, defined as $\left(d-d_{0}\right) /\left(d_{0}\right)$, as a function of $\sin ^{2} \psi$, d and $d_{0}$ being the measured and nominal Ag plane distances in the 111 direction. The slope of the linear function is proportional to the internal stress $\sigma$ (slope $=1 / 2 S_{2} \cdot \sigma$, where $S_{2}$ is an elastic constant that for $\mathrm{Ag}$ reads $3.3 \times 10^{-5} \mathrm{MPa}$ ) that can then be easily derived.

Residual stress analysis indicates compressive stress for $\mathrm{Ag}$ NP grown on $\mathrm{Al}_{2} \mathrm{O}_{3}$. However, the same analysis on a Ag layer deposited on the same substrate, and taken as a reference, reveals a higher residual stress. In Figure S4, the plot of the strain as a function of $\sin ^{2} \psi$ clearly displays a different slope of the linear fits for the NP and the Ag reference sample, resulting in two different strain levels. The strain in the NP is indeed 2.5 times smaller with respect to the strain measured on the Ag layer having the same thickness of the NP. 


\section{Pump Probe Principle}

The all-optical pump-probe technique exploits a laser pulse (pump pulse) to excite a sample at time $\mathrm{t}=0$. A time-delayed probe beam (probe pulse) is then exploited to optically investigate the relaxation dynamics of the excited sample at time-delays $t_{0}, t_{1}, t_{2}, \ldots t_{n}$, the time delays being with respect to the pump beam, see Figure S5. In the present work the relative intensity variation have been measured in both (a) transmission, $\Delta \operatorname{Tr} / \operatorname{Tr}$ and (b) reflection, $\Delta \mathrm{R} / \mathrm{R}$ modes as illustrated in Figure $\mathrm{S} 5$.

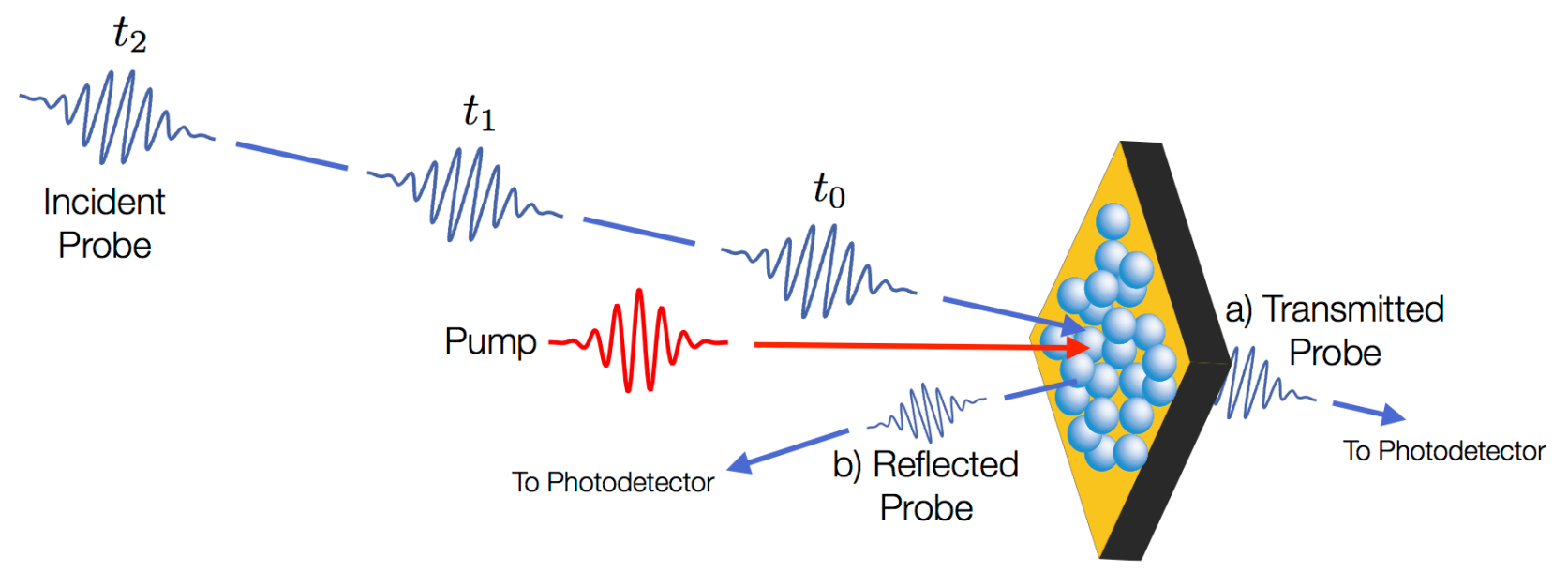

Figure S5. Principle of operation of the optical pump-probe technique. The role of the pump pulse is to excite the sample. The train of pulses of the probe light reveals the modification of the optical properties of the sample as a function of the time delay $t_{i}$ with respect to the pump pulse arrival time. The picture shows the (a) transmission and (b) the reflection configurations. 


\section{Pump Probe Setup}

The measurements set-up is shown in Figure S6. The laser light is provided by the MENLO C-Fiber 780 High Power ASOPS system. The output consists of two synchronized trains of pulses of 100 fs pulse duration at FWHM. The wavelengths of the two beams are $780 \mathrm{~nm}$ (probe) and $1560 \mathrm{~nm}$ (pump). The repetition rates of the cavities are $100 \mathrm{MHz}$ and $100 \mathrm{MHz}$ $+1 \mathrm{KHz}$, respectively. The slight difference in the two lasers repetition rates, at the core of the ASOPS technique, allows obtaining time-delayed probe pulses with respect to the exciting pump pulses without using a moving delay line, i.e. without need of any moving mechanical part. This fact allows measuring relative intensity variation in the order of $10^{-7}$ up to delay times of $10 \mathrm{~ns}$.

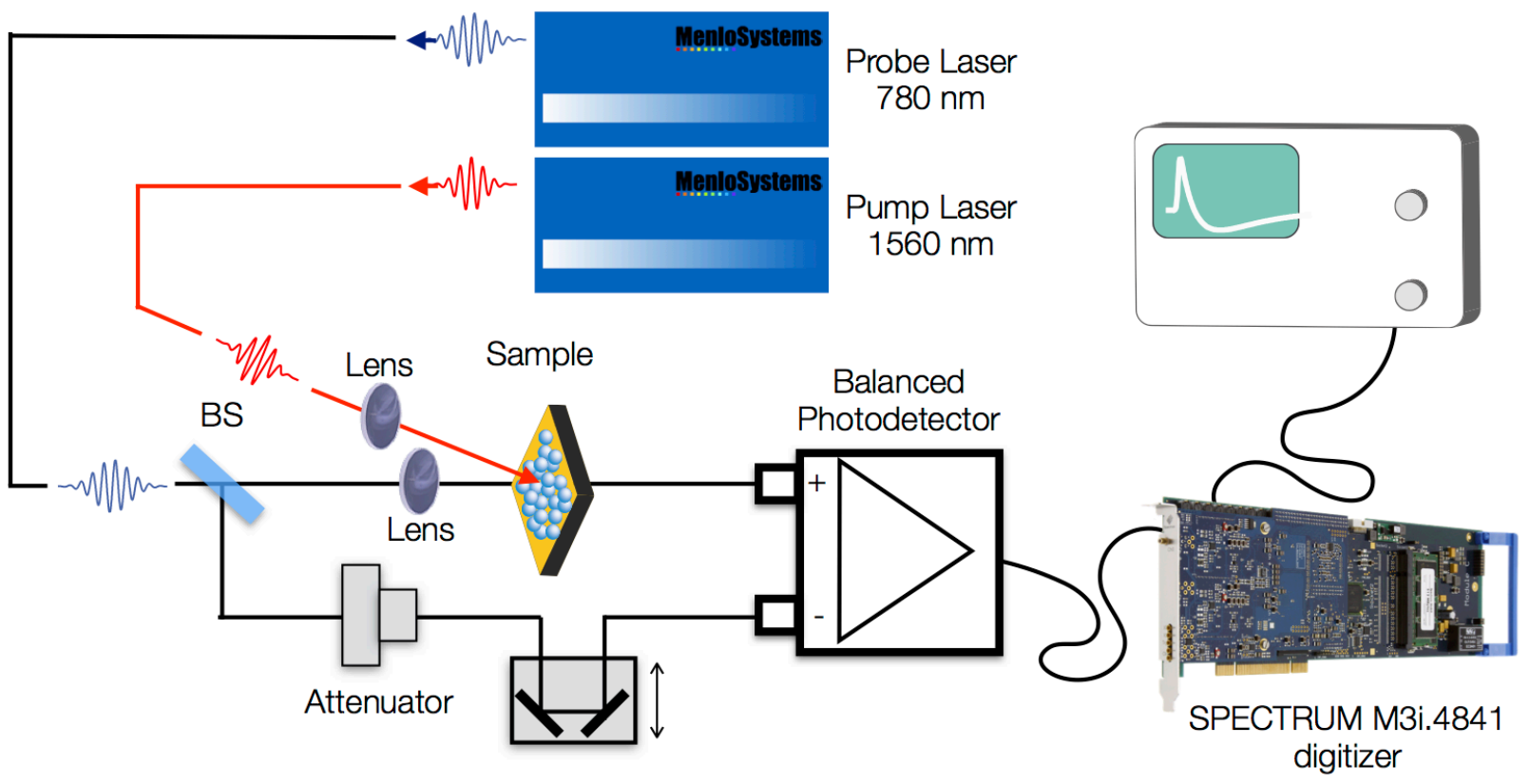

Figure S6. Schematics of the pump-probe optical setup.

The pump and probe beams are focused on the sample through two lenses of focal length $100 \mathrm{~mm}$. The pump diameter at FWHM is $30 \mu \mathrm{m}$; the probe diameter is $15 \mu \mathrm{m}$. Before being focused on the sample, the probe is split into an actual probe beam and a reference beam. Considering the transmission set-up, the variations of the transmitted probe beam, $\Delta \operatorname{Tr}$, is acquired taking the difference between the intensity of the actual probe and the reference beam. The reference beam is conditioned so as to read a null $\Delta \operatorname{Tr}$ when the pump beam is turned off. The reference beam conditioning is achieved modifying (a) its intensity via an optical attenuator and (b) compensating its optical path length with respect to the actual probe via a mechanical delay stage. The difference between the probe and the reference beam is measured via a fast balanced photodetector THORLABS PDB430A, $350 \mathrm{MHz}$ bandwidth. The analog output from the balanced photodetector is digitized via a SPECTRUM M3i.4841 - 16 bit, 105MS/s - acquisition board and finally fed to a computer. 